\title{
A phase I study of the PD-L1 inhibitor, durvalumab, in combination with a PARP inhibitor, olaparib, and a VEGFR1-3 inhibitor, cediranib, in recurrent women's cancers with biomarker analyses
}

\author{
Alexandra S. Zimmer ${ }^{1 *}$ (D), Erin Nichols ${ }^{1,7}$, Ashley Cimino-Mathews ${ }^{2,3}$, Cody Peer ${ }^{4}$, Liang Cao ${ }^{5}$, Min-Jung Lee ${ }^{6}$, \\ Elise C. Kohn', Christina M. Annunziata', Stanley Lipkowitz ${ }^{1}$, Jane B. Trepel ${ }^{6}$, Rajni Sharma ${ }^{3}$, Lekha Mikkilineni', \\ Margaret Gatti-Mays ${ }^{1}$, William D. Figg ${ }^{2}$, Nicole D. Houston ${ }^{1}$ and Jung-Min Lee ${ }^{1}$
}

\begin{abstract}
Background: Strategies to improve activity of immune checkpoint inhibitors are needed. We hypothesized enhanced DNA damage by olaparib, a PARP inhibitor, and reduced VEGF signaling by cediranib, a VEGFR1-3 inhibitor, would complement anti-tumor activity of durvalumab, a PD-L1 inhibitor, and the 3-drug combination would be tolerable.

Methods: This phase 1 study tested the 3-drug combination in a $3+3$ dose escalation. Cediranib was taken intermittently (5 days on/2 days off) at 15 or $20 \mathrm{mg}$ (dose levels 1 and 2, respectively) with durvalumab $1500 \mathrm{mg}$ IV every 4 weeks, and olaparib tablets $300 \mathrm{mg}$ twice daily. The primary end point was the recommended phase 2 dose (RP2D). Response rate, pharmacokinetic (PK), and correlative analyses were secondary endpoints.

Results: Nine patients (7 ovarian/1 endometrial/1 triple negative breast cancers, median 3 prior therapies [2-6]) were treated. Grade 3/4 adverse events include hypertension (1/9), anemia (1/9) and lymphopenia (3/9). No patients experienced dose limiting toxicities. The RP2D is cediranib, $20 \mathrm{mg}$ (5 days on/2 days off) with full doses of durvalumab and olaparib. Four patients had partial responses (44\%) and 3 had stable disease lasting $\geq 6$ months, yielding a $67 \%$ clinical benefit rate. No significant effects on olaparib or cediranib PK parameters from the presence of durvalumab, or the co-administration of cediranib or olaparib were identified. Tumoral PD-L1 expression correlated with clinical benefit but cytokines and peripheral immune subsets did not.
\end{abstract}

Conclusions: The RP2D is tolerable and has preliminary activity in recurrent women's cancers. A phase 2 expansion study is now enrolling for recurrent ovarian cancer patients.

Trial registration: ClinicalTrials.gov identifier: NCT02484404. Registered June 29, 2015.

Keywords: Ovarian cancer, Immune checkpoint inhibitor, PARP inhibitor, VEGF inhibition

\footnotetext{
* Correspondence: alexandra.zimmer@nih.gov

'Women's Malignancies Branch, Center for Cancer Research, National Cancer

Institute, Bethesda, MD, USA

Full list of author information is available at the end of the article
}

(c) The Author(s). 2019 Open Access This article is distributed under the terms of the Creative Commons Attribution 4.0 International License (http://creativecommons.org/licenses/by/4.0/), which permits unrestricted use, distribution, and reproduction in any medium, provided you give appropriate credit to the original author(s) and the source, provide a link to the Creative Commons license, and indicate if changes were made. The Creative Commons Public Domain Dedication waiver (http://creativecommons.org/publicdomain/zero/1.0/) applies to the data made available in this article, unless otherwise stated. 


\section{Introduction}

Immunotherapy has emerged as a major therapeutic modality in oncology. However, the majority of patients with women's cancers do not derive benefit from immune checkpoint blockade monotherapy, creating the need to optimize combination treatment strategies [1].

Data suggest increased DNA damage by radiation or DNA repair inhibitors promotes local antigen release resulting in systemic anti-tumor immune responses [2]. Such neoantigen release and high tumor mutational burden (TMB) are shown to be associated with clinical response to immune checkpoint inhibition in solid tumors [3].

PARP inhibitors (PARPi) are one of the active new drug families in the drug armamentarium for women's cancers and may modulate immune responses. PARPi, such as olaparib and talazoparib were shown to induce PD-L1 expression in breast cancer in vitro and in vivo models [4, 5]. Also, increased DNA damages from PARPi exposure may yield greater TMB, potentially increasing neoantigens, and affect the immune milieu, complementing the clinical benefit of immune checkpoint blockade in subsets of recurrent women's cancer.

Angiogenesis pathways interact with both DNA repair mechanisms and immune activity. Tumor hypoxia induces downregulation of genes involved in DNA repair, e.g., RAD51 and BRCA1, leading to further DNA damages, genomic instability, and cell death [6]. VEGF suppresses lymphocyte trafficking across endothelia into tumor deposits and sites of inflammation to promote vessel growth [7]. Combining inhibition of DNA repair and angiogenesis pathways therefore may modulate the immune response by increasing DNA damage and TMB and by attenuating immunosuppressive microenvironments.

The combination of olaparib and cediranib, a VEGFR1-3 inhibitor, has been demonstrated to be clinically superior to olaparib monotherapy in recurrent platinum-sensitive ovarian cancer [8]. We extended this concept with our hypothesis that reduced VEGF signaling by cediranib and increased DNA damages by olaparib would complement anti-tumor activity of the immune checkpoint inhibitor. Durvalumab (MEDI4736) is a selective, high-affinity human IgG1 monoclonal antibody that blocks PD-L1 binding to PD-1 and CD80, thereby enhancing the function of tumor-directed $\mathrm{T}$ cells [9]. We previously reported the safety data and recommended phase 2 doses (RP2D) of the doublets of durvalumab in combination with olaparib or intermittent cediranib in recurrent women's cancers [10]. We now report safety, RP2D and pharmacokinetics (PK)/pharmacodynamics (PD) findings of the 3-pathway modulation using durvalumab in combination with olaparib and cediranib.

\section{Patients and methods}

\section{Study design and patients}

The trial was approved by the institutional review board of the Center for Cancer Research, National Cancer Institute (ClinicalTrials.gov identifier: NCT02484404). Patients eligible for the study had histologically confirmed advanced breast or gynecologic malignancies, measurable by Response Evaluation Criteria in Solid Tumors (RECIST) v1.1. Prior exposure to PARPi or angiogenesis inhibition was eligible but previous treatment with immune checkpoint blockade was not allowed. Patients had to have controlled hypertension with no more than three antihypertensives, and good end-organ function. Germline BRCA mutation status was requested at baseline. All patients provided written informed consent before enrollment.

Eligible patients received all three drugs in a $3+3$ dose-escalation format as outlined in Table 1. Patients safety was assessed in an ongoing fashion using the Common Terminology Criteria for Adverse Events v4. Response was assessed every two cycles by imaging using RECIST v1.1 criteria. Study treatment was discontinued for progression of disease, intercurrent illness, adverse events (AEs) not recovering to $\leq$ grade 1 within 14 days, or patient withdrawal of consent.

\section{Definitions of dose-limiting toxicity and maximum tolerated dose}

The primary end point was to determine RP2D of the 3drug combination, defined by the maximum tolerated dose (MTD) or the highest protocol-defined dose in the absence of dose-limiting toxicity (DLT). DLT was defined as grade 3 or 4 non-hematologic and grade 4 hematologic AEs related to study medications occurring during the first cycle (28 days). The MTD was defined as the highest dose level at which one or fewer of six patients experienced a DLT. If the observed AE could be specifically attributed to only one of the drugs, that drug was held while the patient continued to receive the drug not associated with the observed AE. Treatment-related serious AEs occurring within 90 days after the last dose of study drugs were reported.

Patients were asked to take blood pressure (BP) twicedaily and given anti-hypertensive medication(s) if two consecutive readings $>140 / 90 \mathrm{mmHg}$. Diarrhea episodes were also recorded in a diary and treated with loperamide per protocol unless associated with immune-mediated colitis.

\section{Pharmacokinetic studies}

Plasma samples were collected at $0.5,1,2,4,8$, and $12 \mathrm{~h}$ after the first dose(s) of olaparib and cediranib and immediately prior to the second dose of olaparib (approximately $12 \mathrm{~h}$ after the first daily dose), on cycle 1 day 1 
Table 1 Dose levels

\begin{tabular}{|c|c|c|c|}
\hline $\begin{array}{l}\text { Dose level } \\
\text { (DL) }\end{array}$ & $\begin{array}{l}\text { Durvalumab } \\
\text { (IV) }\end{array}$ & $\begin{array}{l}\text { Olaparib tablet } \\
\text { (oral) }\end{array}$ & Cediranib (oral) \\
\hline $\mathrm{DL}-1$ & $\begin{array}{l}1500 \mathrm{mg} \text { every } \\
4 \text { weeks }\end{array}$ & $\begin{array}{l}200 \mathrm{mg} \\
\text { twice daily }\end{array}$ & $\begin{array}{l}15 \mathrm{mg} \text { once daily } \\
\text { (5 days on/2 days off) }\end{array}$ \\
\hline $\begin{array}{l}\text { DL } 1 \\
\text { (starting dose) }\end{array}$ & $\begin{array}{l}1500 \mathrm{mg} \text { every } \\
4 \text { weeks }\end{array}$ & $\begin{array}{l}300 \mathrm{mg} \\
\text { twice daily }\end{array}$ & $\begin{array}{l}15 \mathrm{mg} \text { once daily } \\
\text { ( } 5 \text { days on/2 days off) }\end{array}$ \\
\hline DL 2 & $\begin{array}{l}1500 \text { mg every } \\
4 \text { weeks }\end{array}$ & $\begin{array}{l}300 \mathrm{mg} \\
\text { twice daily }\end{array}$ & $\begin{array}{l}20 \text { mg once daily } \\
\text { ( } 5 \text { days on/2 days off) }\end{array}$ \\
\hline
\end{tabular}

before durvalumab administration, and again after durvalumab on cycle 2 day 1 . Plasma was separated and stored at $-80^{\circ} \mathrm{C}$ until measurement. The lower limit of quantitation of the assay is $0.5 \mathrm{ng} / \mathrm{mL}$ for both olaparib and cediranib using previously reported validated methods $[10,11]$. A noncompartmental approach to calculating pharmacokinetics data was employed using Phoenix ${ }^{\oplus}$ WinNonlin v6.4 (Certara Pharsight, Cary, NC).

\section{Archival tissue PD-L1 expression and tumor infiltrating lymphocytes evaluation}

PD-L1 expression on archival tissue was a prespecified exploratory end point. PD-L1 labeling of cancer cells and tumor-infiltrating lymphocytes (TILs) was evaluated in archival tissue samples by immunohistochemistry (IHC). The degree of TIL was assessed as a percentage of stromal and intratumoral space occupied by TIL. Unstained slides of the primary tumor sample from all 9 patients were labeled for PD-L1 by IHC with antibody SP263 (monoclonal rabbit; Ventana Medical Systems Inc., Tucson, AZ) on an automated platform [10]. PD-L1 positivity was defined as labeling of $\geq 1 \%$ carcinoma cells or TIL. The PD-L1 labeling by the carcinoma cells and the TIL was assessed and recorded separately.

\section{Pharmacodynamic studies}

\section{Immune subsets and functional markers analysis}

Peripheral blood mononuclear cells (PBMCs) were collected at baseline (cycle 1 day 1) and on therapy (cycle 1 day 15 and cycle 3 day 1 ). PBMCs were processed within $2 \mathrm{~h}$ and assessed for immune subsets and functional markers using multiparameter flow cytometry as described [12]. CD8+ T cells, CD4+ T cells, Tregs, monocyte subsets and MDSCs were studied. Expression of CTLA-4, T cell immunoglobulin and mucin-domain containing-3 (TIM-3) and PD-1 on CD8+ T cells and Tregs, CD40+ MDSCs, and monocytes HLA-DR and PD-L1 were evaluated as described previously [13]. All analyses were performed using multiparametric flow cytometry (MACSQuant; Miltenyi Biotec, Bergisch Gladbach, Germany), and data were analyzed using FlowJo software v.10.0.7
(FlowJo LLC, Ashland, OR). Flow cytometric data were quantified either as a percentage of a defined cell population, or the median fluorescence intensity (MFI).

\section{Cytokine studies}

Plasma samples collected pre- and on treatment were analyzed by ELISA [14]. The pro-inflammatory cytokines IFN $\gamma$, IL 10, IL 12, IL 2, IL 6, IL 8 and TNF $\alpha$ were examined.

\section{Statistical analyses}

All statistical tests used two-sided significance level 0.05, adjusted for multiple comparisons, using GraphPad Prism software v6.0 (GraphPad Software Inc., La Jolla, $\mathrm{CA})$. Paired $t$-tests were performed in a parametric manner.

\section{Results}

\section{Patient characteristics}

Nine patients were enrolled between June 16, 2016 and January 3,2017 . The majority of the patients had ovarian carcinoma (6/9 [66\%]) and all but one had germline $B R C A$ wild-type recurrent platinum-resistant disease. Baseline patient characteristics are listed in Table 2.

\section{Dose optimization and toxicities}

The dose level 2 was identified as the RP2D, with durvalumab $1500 \mathrm{mg}$ IV every 4 weeks in combination with olaparib $300 \mathrm{mg}$ twice daily and cediranib $20 \mathrm{mg}$ daily ( 5 days on/ 2 days off). No DLT was observed during the treatment with a 3-drug combination. One patient required a one dose level reduction of

Table 2 Baseline characteristics

\begin{tabular}{ll}
\hline Characteristics & N=9 \\
\hline Age, median (range) & 59 year-old (44-73) \\
BRCA mutation status & $1 / 7 / 1$ \\
(germline mutation/wild type/unknown) & \\
Tumor type & $6(2 / 2 / 1 / 1)$ \\
OvCa (HGSOC/Clear cell/Mixed & \\
Mullerian/ Mixed Serous and Endometrioid) & $2 / 5$ \\
Platinum sensitivity in OvCa (sensitive/resistant) & 1 \\
Primary Peritoneal cancer (platinum-resistant) & 1 \\
$\quad$ Endometrial carcinoma (MSI low) & 1 \\
Triple Negative Breast Cancer & $3 / 6 / 0$ \\
ECOG Performance Status (0/1/2) & $2(2-6)$ \\
Number of previous treatments, median (range) & 0 \\
Prior PARPi (n) & 2 \\
Prior bevacizumab (n) & \\
\hline
\end{tabular}

Abbreviations: $N$ number, OvCa ovarian cancer, $H G S O C$ high-grade serous OvCa, MSI microsatellite instability, PARPi poly ADP ribose polymerase inhibitor 
olaparib during cycle 5 , due to recurrent grade 3 anemia. The most common AEs related to treatment were hematologic and gastrointestinal toxicities. All patients had at least one any-grade $\mathrm{AE}$ and those are summarized in Table 3.

Table 3 Treatment related adverse events by maximum grade per patient

\begin{tabular}{|c|c|c|c|c|}
\hline Adverse event & Grade 1 & Grade 2 & Grade 3 & Grade 4 \\
\hline \multicolumn{5}{|l|}{ Hematological } \\
\hline Lymphopenia & 1 & 2 & 3 & 0 \\
\hline Anemia & 2 & 1 & 2 & 0 \\
\hline Thrombocytopenia & 2 & 0 & 0 & 0 \\
\hline Neutropenia & 1 & 1 & 0 & 0 \\
\hline \multicolumn{5}{|l|}{ Gastrointestinal } \\
\hline Anorexia & 2 & 0 & 1 & 0 \\
\hline Nausea & 5 & 0 & 0 & 0 \\
\hline Vomit & 3 & 0 & 0 & 0 \\
\hline Diarrhea & 5 & 0 & 0 & 0 \\
\hline GERD & 0 & 1 & 0 & 0 \\
\hline Dyspepsia & 1 & 1 & 0 & 0 \\
\hline \multicolumn{5}{|l|}{ Endocrinology and Chemistry } \\
\hline Increased creatinine & 1 & 2 & 1 & 0 \\
\hline Hypothyroidism & 1 & 1 & 0 & 0 \\
\hline Proteinuria & 1 & 0 & 0 & 0 \\
\hline Increase ALT/AST & 4 & 0 & 0 & 0 \\
\hline Increase Alkaline Phosphatase & 2 & 0 & 0 & 0 \\
\hline \multicolumn{5}{|l|}{ Cardiovascular } \\
\hline Hypertension & 0 & 3 & 1 & 0 \\
\hline Syncope & 0 & 0 & $1^{a}$ & 0 \\
\hline DVT & 0 & $1^{b}$ & 0 & 0 \\
\hline \multicolumn{5}{|l|}{ Other } \\
\hline Fatigue & 6 & 2 & 0 & 0 \\
\hline Dyspnea & 1 & 0 & 0 & 0 \\
\hline Headache & 1 & 0 & 0 & 0 \\
\hline Arthralgia & 2 & 0 & 0 & 0 \\
\hline Dizziness & 2 & 0 & 0 & 0 \\
\hline Gastric hemorrhage & 0 & 1 & 0 & 0 \\
\hline Hoarseness & 1 & 0 & 0 & 0 \\
\hline
\end{tabular}

Anemia occurred in 5 of 9 patients, one with grade 3 anemia required olaparib dose reduction. One patient was taken off study treatment for extensive progression of disease after 3 cycles of treatment and developed multifactorial causes for renal failure, grade 3 creatinine elevation and grade 3 anemia at the time. This patient also developed a new-onset DVT in lower extremity after cycle 3 of treatment, thus cediranib was discontinued but other two drugs were continued

Abbreviations: GERD gastroesophageal reflux disease, AST aspartate aminotransferase, $A L T$ alanine aminotransferase, DVT deep venous thrombosis anlikely related to study drugs - determined to be a non-drug related vasovagal episode after extensive cardiovascular investigation including brain imaging

${ }^{\text {b}}$ Possibly related to cediranib and disease, cediranib was discontinued after grade 2 DVT event but durvalumab and olaparib were continued

\section{Clinical activity}

The objective response rate was $44 \%$ (4/9) with all partial responses (PRs), lasting a median of 8.5 months [range 7 to 26 months]. One patient with PR was still receiving the study treatment at the time of data cutoff (October 25, 2018), with 26+ months of continuous treatment. Three patients (33\%) had stable disease (SD), 2 of them lasting 19 and 21 months, respectively. The clinical benefit rate, defined herein as complete response $[C R]+P R+S D \geq 6$ months, was $67 \%(6 / 9)$. Changes from baseline in tumor size and duration of response are shown in Fig. 1.

\section{PK studies}

No clinically significant effects on olaparib PK from the presence of durvalumab, or the co-administration of cediranib were identified. Olaparib exposures were comparable before and after durvalumab, either alone or with cediranib, and clearance and volume of distribution were unchanged. Similarly, with the intermittent dosing schedule for cediranib (5 days on/2 days off), no statistically significant changes in cediranib PK parameters were caused by the presence of durvalumab, olaparib, and durvalumab plus olaparib (Additional file 1: Figure S1).

\section{Archival tissue TIL infiltration and PD-L1 expression}

Some degrees of TIL infiltration were present in all tumors: focal $(<5 \%$ tumoral TIL; $3 / 9)$, moderate $(5-50 \%$ tumoral TIL; $5 / 9)$ and brisk TIL infiltration ( $\geq 50 \%$ tumoral TIL; $1 / 9)$. The majority of tumors (7/9) had PDL1 positive carcinoma cells, and most cases (8/9) contained PD-L1 positive TIL. All seven patients with PDL1 positive carcinoma cells had SD or PR as best response, whereas two patients with PD-L1 negative carcinoma cells had PD $(p=0.03)$ (Additional file 2: Figure S2 and Additional file 5: Table S1). The two tumors with no carcinoma cell PD-L1 labeling, contained PD-L1 positive TIL. However, there was no statistically significant association between the degree of TIL infiltrate, the degree of TIL PD-L1 labeling, or the degree of carcinoma cell PD-L1 labeling and the duration of response.

\section{PD studies}

\section{Immune subsets and functional markers analysis}

PBMCs of all patients $(n=9)$ were evaluated for immune subsets and functional markers. A transient increase in PD-L1 expression on total CD14+ monocytes was observed on cycle 1 day 15 (median MFI 1.8 [baseline] vs 3.0 [cycle 1 day 15]; $p<0.0001$ ) which was not sustained on cycle 3 day 1 (Additional file 3: Figure S3B). Other innate and adaptive immune cells and functional markers were analyzed and did not demonstrate significant changes after treatment. 


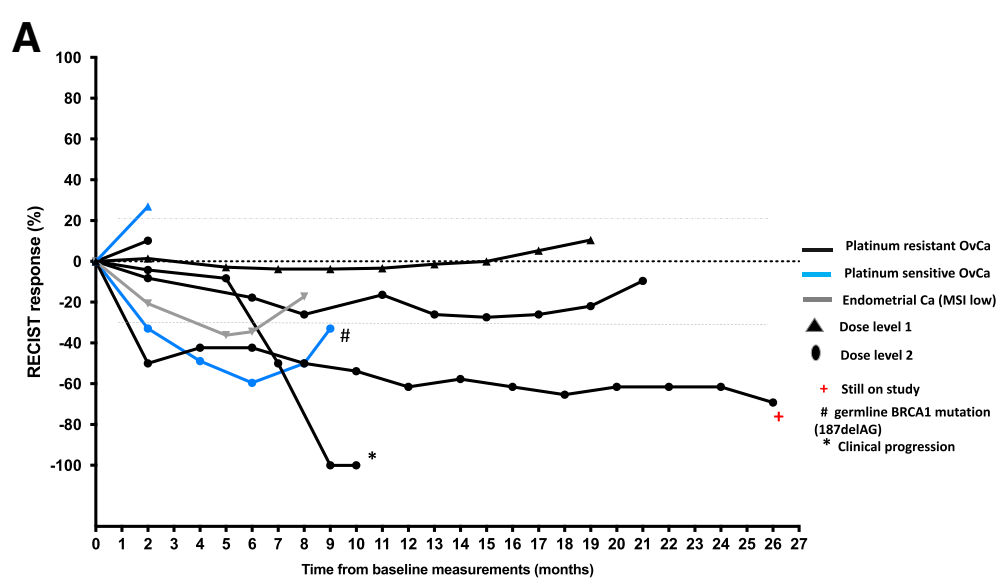

B
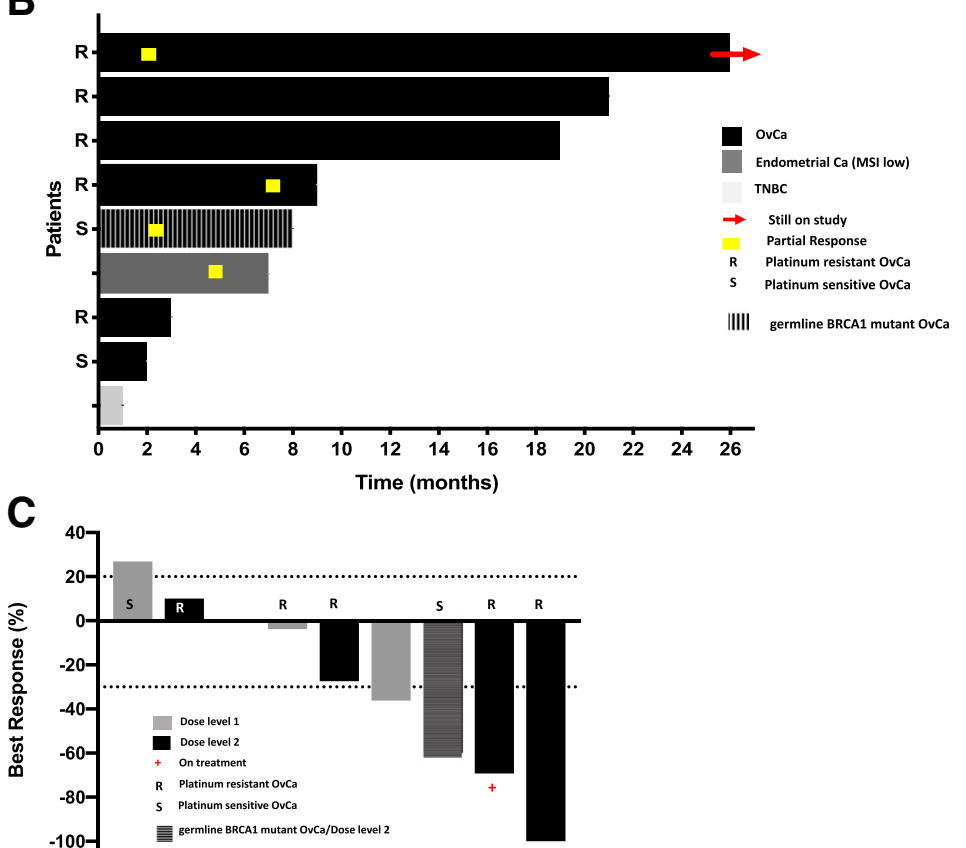

Fig. 1 Changes in tumor size and duration on the treatment. a Changes in tumor size on the study treatment. b Duration in the study. c Best response. One patient with TNBC had clinical progressive disease before first response evaluation imaging and is represented as zero (0) in a and b. Abbreviations: S: platinum-sensitive recurrent ovarian cancer, R: platinum-resistant recurrent ovarian cancer. OvCa: ovarian cancer. TNBC: triple negative breast cancer

\section{Cytokines}

Changes in circulating proinflammatory cytokines (IFN $\gamma$, TNF $\alpha$, IL2, IL6, IL8, IL10, and IL12) before and after treatment were evaluated. The median values of circulating cytokines were not statistically different between baseline and cycle 1 day 15 or cycle 3 day 1 (Additional file 4: Figure S4).

\section{Discussion}

This is the first reported 3-drug combination therapy of immune checkpoint blockade with a PARPi and a VEGFR inhibitor. This study established the RP2D of 3-drug combination and demonstrates preliminary evidence of activity in heavily pretreated recurrent gynecologic cancer patients, particularly in ovarian cancer. We previously reported durvalumab with an intermittent cediranib schedule had greater tolerability without attenuating clinical benefit observed in the daily schedule [10]. We now observe a full dose olaparib can be added to that combination in a safe manner using prospective toxicity mitigation approaches. Unlike our prior observations where durvalumab affected Cmax and clearance of daily cediranib [10], PK parameters of cediranib and olaparib were not affected by the presence of durvalumab in this 3drug combination. 
The current study presents durable long-term activity and safety outcome of the 3-pathway modulation with a median of 8 months follow-up (range 1 to $26+$ months) and a RR worthy of further investigation. Single agent olaparib has limited activity (4\%) in heavily pretreated $B R C A$ wild-type patients with platinum-resistant ovarian cancer [15]. Cediranib monotherapy also demonstrated a RR of $17 \%$ and a median PFS of 5.2 months in patients with platinum-resistant ovarian cancer, treated in the 2nd or 3rd line setting [16]. However, our data should be interpreted with a caution due to its small sample size and phase 1 single-arm dose escalation design, thereby preventing direct comparison of the combination with either drug alone.

There are limited published data on anti-tumor activity and safety of PD-1/PD-L1 blockade combinations with a PARPi or VEGF/VEGFR inhibition in women's cancer. Preliminary results from two PARPi and PD-1/PD-L1 blockade combination trials were presented showing early clinical activity in subsets of recurrent ovarian cancer; 25\% RR of niraparib and pembrolizumab in platinum-resistant ovarian cancer [17] and 72\% RR of durvalumab and olaparib in germline $B R C A$ mutant platinum-sensitive ovarian cancer patients [18]. Our group reported, at the 2018 European Society for Medical Oncology (ESMO) meeting, a $14 \% \mathrm{RR}$ and $37 \%$ clinical benefit rate (as defined in Methods) of the combination durvalumab and olaparib, both in full doses, in heavily pretreated ovarian cancer patients, predominantly composed of $B R C A$ wild-type and platinum-resistant disease [19]. A RR of $25 \%$ with niraparib and pembrolizumab was shown in women with platinum-resistant ovarian cancer [17], and in germline $B R C A$ mutant platinum-sensitive ovarian cancer, a $72 \%$ RR with durvalumab and olaparib [18]. The single arm phase II study of bevacizumab and nivolumab in recurrent ovarian cancer presented at ESMO 2018 reported 16.7\% RR and $55.3 \%$ CBR (defined by $\mathrm{CR}+\mathrm{PR}+\mathrm{SD} \geq 6$ months), with a median PFS of 5.3 months in platinum-resistant patients (18 patients) and $40 \% \mathrm{RR}$ and $75 \%$ CBR, with a median PFS of 9.4 months in those with platinum-sensitive disease (20 patients), respectively [20]. These findings suggest a modest benefit of addition of either PARPi or bevacizumab to PD-1/PD-L1 blockade in germline BRCA wildtype ovarian cancer patients, warranting further investigation of our 3-drug combination strategy. Separately, phase 3 randomized trials of 3-pathway modulation (immune checkpoint inhibitor in combination with PARPi and VEGF inhibition) are now being investigated in the frontline setting for ovarian cancer treatment (e.g., avelumab (PD-L1 inhibitor) + talazoparib (PARPi) + bevacizumab, ClinicalTrials.gov identifier: NCT03642132).

Defining predictive biomarkers of immune checkpoint inhibitor-based combination therapy is a major challenge. Our study suggests that presence of PD-L1 positive carcinoma cells in archival tissues may be associated with clinical benefit. In contrast, peripheral immune subsets analysis showed a transient increase of PD-L1 expression on total CD14+ monocytes after 2 weeks treatment of olaparib and cediranib although it did not last after 2 months treatment. An association of tumoral PD-L1 expression and response to immune checkpoint inhibitor therapy has been reported in ovarian carcinoma [21] and triple negative breast cancer (TNBC) [22]. The correlation of clinical benefit and expression of PD-L1 using archival tissues needs further validation, particularly in recurrent women's cancers given preanalytical variabilities (e.g., different IHC techniques, score systems, different antibodies $[23,24])$ and possible dynamic changes of PD-L1 expression affected by prior therapies [25]. The present study is now being expanded to a phase 2 study for patients with recurrent ovarian cancer. We will prospectively examine the association of PD-L1 induction and TMB by PARPi with clinical response to immune checkpoint blockade using fresh core biopsies collected before and on-treatment.

In summary, our study demonstrates that the 3-drug combination is tolerable and active in heavily pretreated recurrent gynecologic cancer patients without germline $B R C A$ mutation. This response may be associated with carcinoma cell PD-L1 labeling, but this requires further validation. The preliminary activity findings warrant further investigation and a single-arm phase II expansion study is now open to accrual for recurrent ovarian cancer patients (ClinicalTrials.gov identifier: NCT02484404).

\section{Additional files}

Additional file 1: Figure S1. Pharmacokinetics effects of durvalumab on olaparib and cediranib. (A-B) Durvalumab did not affect olaparib PK Cmax or AUC. (C-D) The presence of durvalumab did not significantly affect cediranib PK. One patient's PK data is missing due to no sample collection. One patient (red dot) showed abnormally low plasma concentrations that led to higher than normal CLss/F, possibly due to food effect on absorption. Abbreviations: $A \cup C_{I_{N F}}$ : area under the plasma concentration $v$. time curve from time zero to infinity. AUC/D: Area under the plasma concentration $v$. time curve normalized to dose. AUC TAU: AUC for the dosing interval for steady-state kinetics after durvalumab; $12 \mathrm{~h}$ for olaparib, $24 \mathrm{~h}$ for cediranib (PPTX $161 \mathrm{~kb}$ )

Additional file 2: Figure S2. Tumor infiltrating lymphocytes (TIL) and PD-L1 analysis by immunohistochemistry. (A-B) Patient B04 had a PR of 9 months duration; her primary HGSOC (arrow) showed PD-L1 positivity in the carcinoma cells, as well as within the TIL (star) ( $\times 200)$. (C-D) Patient B09 experienced PD; her primary TNBC (arrow) did not display any PD-L1 labeling, and there were minimal TIL $(<5 \%)$ within the tumor bed. Abbreviations: PR: partial response, HGSOC: high grade serous ovarian carcinoma, TIL: tumor infiltrating lymphocytes, TNBC: triple negative breast cancer (PPTX $9168 \mathrm{~kb}$ )

Additional file 3: Figure S3. Peripheral immune subsets and functional markers. (A) CD8/CD4 ratio. (B) PD-L1 expression on total C14+ monocytes. Open dots: germinative BRCA mutated cases. Abbreviations: MFI: median fluorescence intensity. (PPTX $95 \mathrm{~kb}$ ) 
Additional file 4: Figure S4. Proinflammatory cytokines analysis. Plasma levels of pro-inflammatory cytokines (IFN $\gamma$, TNFa, IL 2, IL 6, IL 8 IL 10, and IL 12) were not changed significantly by the treatment. (PPTX $189 \mathrm{~kb}$ )

Additional file 5: Table S1. Pathologic characteristics and immune correlates. (DOCX $15 \mathrm{~kb})$

\begin{abstract}
AE: Adverse event; CBR: Clinical benefit rate; CR: Complete response; DLT: Dose-limiting toxicity; IHC: Immunohistochemistry; MDSCs: Myeloidderived suppressor cells; MFI: Median fluorescence intensity; MMR: Mismatch repair; MSI: Microsatellite instability; MTD: Maximum tolerated dose; PARPinhibitor: Poly ADP ribose polymerase inhibitor; PBMCs: Peripheral blood mononuclear cells; PK: Pharmacokinetic; PR: Partial response; RP2D: Recommended phase 2 dose; RR: Response rate; SD: Stable disease; TIL: Tumor infiltrating lymphocytes; TMB: Tumor mutational burden; TNBC: Triple-negative breast cancer; Treg: Regulatory T cells; VEGFR 1-3 inhibitor: Vascular endothelial growth factor receptors 1-3 inhibitor
\end{abstract}

\section{Acknowledgements}

We thank Drs. Zia, Parker, Minasian and Nunes, Ekwede RN, and Ms. Gomez for their contributions in clinic.

\section{Authors' contributions}

JML and ECK designed and developed the study. ASZ, EN, ECK, CMA, SL, LM, MGM, NDH and JML participated in study development and patient care. ASZ, ACM, CP, LC, MJL, JBT, RS, WDF and JML performed experiments, acquired and/or analyzed the data. ASZ and JML wrote the manuscript. All authors read and approved the final manuscript.

\section{Funding}

This research was supported by the Intramural Research Program of the National Cancer Institute (NCI), Center for Cancer Research. This project has been funded in whole or in part with federal funds from the National Cancer Institute, National Institutes of Health, under Contract No. HHSN261200800001E. The content of this publication does not necessarily reflect the views or policies of the Department of Health and Human Services, nor does mention of trade names, commercial products, or organizations imply endorsement by the U.S. Government. Durvalumab, olaparib and cediranib were supplied to the Center for Cancer Research, $\mathrm{NCl}$ under a Cooperative Research and Development Agreement between the Center for Cancer Research/NCI and AstraZeneca/Medlmmune.

\section{Availability of data and materials}

All data generated or analysed during this study are included in this published article (and its additional files).

\section{Ethics approval and consent to participate}

This clinical trial was approved by the NIH IRB.

\section{Consent for publication}

All patients enrolled in the clinical trial signed Informed Consent.

\section{Competing interests}

The authors declare that they have no competing interests.

\section{Author details}

${ }^{1}$ Women's Malignancies Branch, Center for Cancer Research, National Cancer Institute, Bethesda, MD, USA. ${ }^{2}$ Johns Hopkins Hospital Department of Pathology, Baltimore, MD, USA. ${ }^{3}$ Johns Hopkins Hospital Department of Oncology, Baltimore, MD, USA. ${ }^{4}$ Genitourinary Malignancies Branch, National Cancer Institute, Bethesda, MD, USA. ${ }^{5}$ Genetics Branch, National Cancer Institute, Bethesda, MD, USA. ${ }^{6}$ Developmental Therapeutics Branch, Center for Cancer Research, National Cancer Institute, Bethesda, MD, USA. ${ }^{7}$ Clinical Monitoring Research Program Directorate, Frederick National Laboratory for Cancer Research sponsored by the National Cancer Institute, Bethesda, MD, USA.
Received: 6 May 2019 Accepted: 16 July 2019

Published online: 25 July 2019

\section{References}

1. J-m L, Ivy SP, Kohn EC. Challenges and opportunities for immunotherapies in gynecologic cancers. Oncology New York. 2016;30(1):67-9.

2. Mouw KW, Goldberg MS, Konstantinopoulos PA, D'Andrea AD. DNA damage and repair biomarkers of immunotherapy response. Cancer Discov. 2017;7(7):675-93.

3. Hellmann MD, Nathanson T, Rizvi H, et al. Genomic features of response to combination immunotherapy in patients with advanced non-small-cell lung Cancer. Cancer Cell. 2018;33(5):843-+

4. Jiao S, Xia W, Yamaguchi H, et al. PARP inhibitor upregulates PD-L1 expression and enhances Cancer-associated immunosuppression. Clin Cancer Res. 2017;23(14):3711-20.

5. Sun C, Fang Y, Yin J, et al. Rational combination therapy with PARP and MEK inhibitors capitalizes on therapeutic liabilities in RAS mutant cancers. Sci Transl Med. 2017:9(392).

6. Hegan DC, Lu Y, Stachelek GC, Crosby ME, Bindra RS, Glazer PM. Inhibition of poly (ADP-ribose) polymerase down-regulates BRCA1 and RAD51 in a pathway mediated by E2F4 and p130. Proc Natl Acad Sci U S A. 2010;107(5):2201-6.

7. Kandalaft LE, Motz GT, Busch J, Coukos G. Angiogenesis and the tumor vasculature as antitumor immune modulators: the role of vascular endothelial growth factor and endothelin. Cancer Immunol Immunother. 2011;344:129-48.

8. Liu JF, Barry WT, Birrer M, et al. Combination cediranib and olaparib versus olaparib alone for women with recurrent platinum-sensitive ovarian cancer: a randomised phase 2 study. Lancet Oncol. 2014;15(11):1207-14.

9. Munoz-Unceta N, Burgueno I, Jimenez E, Paz-Ares L. Durvalumab in NSCLC: latest evidence and clinical potential. Ther Adv Med Oncol. 2018;10: 1758835918804151.

10. Lee J-M, Cimino-Mathews A, Peer CJ, et al. Safety and clinical activity of the programmed death-ligand 1 inhibitor Durvalumab in combination with poly (ADP-ribose) polymerase inhibitor Olaparib or vascular endothelial growth factor receptor 1-3 inhibitor Cediranib in Women's cancers: a doseescalation, phase I study. J Clin Oncol. 2017:35(19):2193-+.

11. Roth J, Peer CJ, Mannargudi B, et al. A sensitive and robust ultra HPLC assay with tandem mass spectrometric detection for the quantitation of the PARP inhibitor Olaparib (AZD2281) in human plasma for pharmacokinetic application. Chromatography. 2014:(1):82-95.

12. Rajan A, Carter CA, Berman A, et al. Cixutumumab for patients with recurrent or refractory advanced thymic epithelial tumours: a multicentre, open-label, phase 2 trial. Lancet Oncol. 2014;15(2):191-200.

13. Tomita $Y$, Lee $M J$, Lee $S$, et al. The interplay of epigenetic therapy and immunity in locally recurrent or metastatic estrogen receptor-positive breast cancer: correlative analysis of ENCORE 301, a randomized, placebocontrolled phase II trial of exemestane with or without entinostat. Oncoimmunology. 2016;5(11):e1219008.

14. Lee J-M, Trepel JB, Choyke P, et al. CECS and IL-8 have prognostic and predictive utility in patients with recurrent platinum-sensitive ovarian cancer: biomarker correlates from the randomized phase-2 trial of olaparib and cediranib compared with olaparib in recurrent platinum-sensitive ovarian cancer. Front Oncol. 2015:5:123.

15. Gelmon KA, Tischkowitz M, Mackay H, et al. Olaparib in patients with recurrent high-grade serous or poorly differentiated ovarian carcinoma or triple-negative breast cancer: a phase 2, multicentre, open-label, nonrandomised study. Lancet Oncol. 2011;12(9):852-61.

16. Matulonis UA, Berlin S, Ivy $P$, et al. Cediranib, an Oral inhibitor of vascular endothelial growth factor receptor kinases, is an active drug in recurrent epithelial ovarian, fallopian tube, and peritoneal Cancer. J Clin Oncol. 2009; 27(33):5601-6.

17. Konstantinopoulos $P$, Munster $P$, Forero-Torez A, et al. Topacio: preliminary activity and safety in patients (pts) with platinum-resistant ovarian cancer (PROC) in a phase $1 / 2$ study of niraparib in combination with pembrolizumab. Gynecol Oncol. 2018;149:246.

18. Drew $Y$, de Jonge $M$, Hong $S$, et al. An open-label, phase II basket study of olaparib and durvalumab (MEDIOLA): results in germline BRCA-mutated (gBRCAm) platinum-sensitive relapsed (PSR) ovarian cancer (OC). Gynecol Oncol. 2018;149:246-7. 
19. Lee J, Annunziata C, Houston N, et al. A phase 2 study of durvalumab, a PDL1 inhibitor and olaparib in recurrent ovarian cancer (OvCa). Ann Oncol. 2018;29:332-58.

20. Liu J, Herold C, Luo W, et al. A phase 2 trial of combination nivolumab and bevacizumab in recurrent ovarian cancer. Ann Oncol. 2018;29.

21. Varga A, Piha-Paul SA, Ott PA, et al. Pembrolizumab in patients (pts) with PD-L1-positive (PD-L1(+)) advanced ovarian cancer: updated analysis of KEYNOTE-028. J Clin Oncol. 2017;35.

22. Schmid P, Adams S, Rugo HS, et al. Atezolizumab and nab-paclitaxel in advanced triple-negative breast Cancer. N Engl J Med. 2018.

23. Liu D, Wang S, Bindeman W. Clinical applications of PD-L1 bioassays for cancer immunotherapy. J Hematol Oncol. 2017;10:110.

24. Hirsch FR, McElhinny A, Stanforth D, et al. PD-L1 immunohistochemistry assays for lung Cancer: results from phase 1 of the blueprint PD-L1 IHC assay comparison project. J Thorac Oncol. 2017:12(2):208-22.

25. Vilain RE, Menzies AM, Wilmott JS, et al. Dynamic changes in PD-L1 expression and immune infiltrates early during treatment predict response to PD-1 blockade in melanoma. Clin Cancer Res. 2017;23(17):5024-33.

\section{Publisher's Note}

Springer Nature remains neutral with regard to jurisdictional claims in published maps and institutional affiliations.

Ready to submit your research? Choose BMC and benefit from:

- fast, convenient online submission

- thorough peer review by experienced researchers in your field

- rapid publication on acceptance

- support for research data, including large and complex data types

- gold Open Access which fosters wider collaboration and increased citations

- maximum visibility for your research: over $100 \mathrm{M}$ website views per year

At $\mathrm{BMC}$, research is always in progress.

Learn more biomedcentral.com/submissions 